\title{
The Impact of COVID-19 on National Clinical Trials Network Breast Cancer Trials
}

\author{
Amara Ndumele ${ }^{1} \cdot$ Ko Un Park ${ }^{2}$ iD \\ Accepted: 22 April 2021 / Published online: 12 May 2021 \\ (C) The Author(s), under exclusive licence to Springer Science+Business Media, LLC, part of Springer Nature 2021
}

\begin{abstract}
Purpose of review The COVID-19 pandemic has had a devastating impact on virtually all aspects of the healthcare system, including oncology clinical trials. The purpose of this review is to describe the impact of the pandemic on national breast cancer clinical trials. Recent findings Of the 61 breast cancer-specific cooperative group breast cancer clinical trials open to accrual during the pandemic, $32 \%$ of them received supplementary tailored guidance regarding current and foreseeable challenges for clinical sites and investigators due to COVID-19. Many clinical trial sites reported decreases in enrollment and accrual, drastic disruptions to protocol, and challenges related to research infrastructure, staff burden, and assuring social distancing.

Summary The challenges early in pandemic led to many operational changes in clinical trials, including use of electronic consent for enrollment, telemedicine visits, and mail order pharmacy. The pandemic highlighted aspects of breast cancer clinical trial that could be modernized while maintaining research integrity.
\end{abstract}

Keywords Breast cancer · Clinical trial · COVID-19 · Telemedicine

\section{Introduction}

As of January 2, 2021, the World Health Organization reported over 82 million cases and 1.8 million deaths due to SARS$\mathrm{CoV}-2$ virus [1]. With over 5 million confirmed cases in the USA, this pandemic has had a devastating impact on virtually all aspects of the healthcare system, including oncology care $[1,2]$. Especially during early phases of the pandemic, guidelines focused on managing and containing COVID-19 as a priority $[3,4]$. Thus, hospitals had to conserve adequate personal protective equipment and resources to manage the influx of COVID-19 patients. Oncology clinical research is one particular area that was affected by this necessary prioritization.

This article is part of the Topical Collection on Breast Cancer Management during the COVID-19 Pandemic

Ko Un Park

Koun.park@osumc.edu

1 College of Medicine, The Ohio State University, Columbus, OH, USA

2 Division of Surgical Oncology, Department of Surgery, The Ohio State University Wexner Medical Center and James Comprehensive Cancer Center, Columbus, OH, USA
There are over 16.9 million cancer survivors in the USA, and many of these patients have benefited from enrollment in clinical trials [5]. During the initial phase of the pandemic in the USA, from March to April 2020, according to the ClinicalTrials.gov website, 1052 clinical trials were suspended with 905 reported suspension primarily due to the COVID-19 pandemic [6]. Over 200 of those studies were specific to interventional oncology studies [7]. Furthermore, there was a $41.8 \%$ reduction in recruiting participants for oncologic clinical trials compared to this time last year [8]. The primarily purpose of this review is to [1] describe the impact the COVID-19 pandemic has had on ongoing breast clinical trials funded through the National Cancer Institute's National Clinical Trials Network (NCTN), [2] explore the various challenges and recommendations made by regulatory agencies and research centers to address them, and [3] discuss the future concerns and opportunities for modernizing clinical trials.

\section{NCTN}

The National Clinical Trials Network was established in order to coordinate and support cancer clinical trials at over 2200 sites in the USA and internationally. The NCTN consists of 
four adults' groups, one pediatric group, and includes the National Cancer Institute of Canada-Clinical Trials Group (NTIC-CTG). The five US Network Groups are the Alliance for Clinical Trials in Oncology, Eastern Cooperative Oncology Group-American College of Radiology Imaging Network (ECOG-ACRIN) Cancer Research Group, NRG Oncology, Southwest Oncology Group (SWOG) Cancer Research Network, and the Children's Oncology Group (COG). The NCTN system has a centralized institutional review board (IRB) and cancer trials support unit (CTSU) as part of the Cancer Therapy Evaluation Program (CTEP), Imaging and Radiation Oncology Core (IROC) group, and a unified data management system. The NCI provides oversight through scientific steering committees that establish strategic priorities for the network and coordinate trials among the cooperative groups $[9,10]$.

\section{Impact on Accrual and Enrollment}

We queried the US National Library of Medicine's website ClinicalTrials.gov, CTSU, and specific cooperative group websites to identify NCTN breast clinical trials that were impacted from March 1 to August 30, 2020. The reason for the suspension, protocol deviation, or amendment was recorded as reported by the studies.

As of August 2020, there were a total of 61 breast cancerspecific NCTN clinical trials open to accrual. Despite NCTN's research infrastructure and collaboration, the pandemic has negatively impacted many aspects of ongoing clinical trials. At the 1st Virtual Joint Meeting of NCI Board of Scientific Advisors and National Cancer Advisory Board, from February to March 2020, there was a $44 \%$ and $42 \%$ decrease in accrual for intervention and screening studies, respectively, amongst all cancer sites and groups within the NCTN network [11]. Many trials reported drastic disruptions to protocol and challenges related to patient safety. NCTNaffiliated institutions anticipated a temporarily drop accrual to most studies $[12,13]$. In an analysis of SWOG clinical trial enrollment, from March 22 until the end of the study period, weekly accrual did not exceed 74 patients [14]. This was partly due to the volume of COVID-19 patients overwhelming the healthcare system's ability to provide care for cancer study participants and healthcare systems limiting accrual for nontherapeutic studies. Cumulative COVID-19 infections neared 1 million in the USA during this period. The impact of COVID-19 overwhelming the healthcare system is highlighted most drastically in New York City, wherein over 50,000 hospitalizations were associated with COVID-19 as of May 16, 2020 [15, 16]. Perlmutter Cancer Center, NYU Langone Health, in New York temporarily stopped enrolling patients on inpatient trials and early-phase trials lacking clear clinical benefit [17]. On the contrary, several institutions did not close trial enrollment, including the NIH clinical center, and trial accrual continued where research staff could treat patients safely $[16,18]$.

\section{Adjustments in Regulatory Guidance}

The Food and Drug Administration (FDA), the European Commission, and the Italian Medicines Agency issued special guidance for the conduction of clinical trials during the COVID-19 emergency. The FDA provided a 35-page document of recommendations to assist industry, investigators, and institutional review boards in assuring the safety of human subjects, upholding compliance with good clinical practice, and reducing risks to integrity of clinical trials [19-21]. Six days after the WHO formally declared COVID-19 a pandemic, the National Institutes of Health (NIH) released guidance for NIH-funded clinical trials of which the primary concern was the "safety and welfare of human subject participants and research staff." [22, 23] NIH encouraged investigators to consult associated IRBs on issues related but not limited to virtual and in-person study visits, large gatherings, travel, and flexibilities for laboratory tests. Following suit, the NCI reported a list of adjustments to NCTN clinical trials, including extended deadlines for applications, flexibility regarding project extensions, accommodations for unanticipated costs, and extensions of post-award reporting requirements and expenditures of funds [22].

According to data from the CTSU website, $32 \%$ of NCTN breast cancer-specific trials $(n=61)$ received supplementary tailored guidance regarding current and foreseeable challenges for clinical sites and investigators due to COVID-19. Centralizing and streamlining key elements in trials became an important mechanism to continue enrollment, maintain collection of vital information, and overcome resource constraints [4]. The NCTN cooperative groups dedicated additional COVID-19-specific sections on their websites to provide guidance for informed consent, pre-study testing, and patient assessments that may require additional in-person visit. The following three NCTN trials highlight the various ways that current clinical trials adjusted the regulatory guidance due to the pandemic.

The SWOG study, S1418/BR006, is a randomized phase III trial evaluating the efficacy and safety of adjuvant pembrolizumab for triple receptor-negative breast cancer (TNBC). Since S1418 is conducted under an investigation new drug (IND), pembrolizumab must be administered at an approved/NCI registered site participating in the S1418 protocol. If patients were not able to travel to participating site or if the site-specific clinics had to restrict patients from entering the clinic, there were limited workarounds to provide the necessary treatment drug to the patients. Treatment delays of up to 84 days were allowable per protocol, with appropriate 
documentation. However, treatment delays of longer than 84 days resulted in the patient being removed from the protocol treatment permanently [24].

Another NCTN trial, EA 1131, did not have the same restrictions of an IND trial allowing for modifications to the study protocol to accommodate COVID-19-related restrictions. The investigators of EA 1131_ "Randomized Phase III Post-Operative Trial of Platinum Based Chemotherapy vs. Observation in Patients with Residual Triple- Negative Basal-Like Breast Cancer following Neoadjuvant Chemotherapy" - issued a memorandum allowing a number of modifications due to the pandemic. These included provisions that informed consent could be conducted virtually, treatment initiation could be delayed by an additional 4 weeks beyond allocated time from randomization, patients could have labs and treatment locally, toxicity assessments could be performed over the phone or via telehealth, and that in-person clinic visits were not mandatory for enrollment. These changes provided an avenue to explore feasibility of implementing telemedicine in clinical trial protocols long term $[25,26]$.

Nontherapeutic trials were often the first to temporarily suspend enrollment as hospitals had to prioritize limiting the movement of patients to the facility during the early pandemic. For instance, the ECOG TMIST study (Tomosynthesis Mammographic Imaging Screening Trial) remained open for accrual nationally. This is a randomized breast screening trial designed to compare the performance between digital mammography (2-D) and tomosynthesis mammography (3-D) in finding breast cancer in women who have no symptoms [27]. The decision for active participation was left to individual sites and some sites had to suspend non-urgent health care services, such as screening mammography and other nonessential research operations [28].

Although regulatory and overseeing organizations provided comprehensive guidance and communication, individual investigators and sponsors had to consider specific ongoing recommendations that may differ by state, country, or region. The impact of varying regional and national approaches to COVID19 is best highlighted in the ASCOLT trial (ClinicalTrial.gov NCT00565708). A multicenter phase 3 trial, ASCOLT is a study evaluating aspirin vs placebo after standard adjuvant therapy for high-risk colorectal cancer. This international trial has a total of 41 sites, majority of which are in Asian countries. Quick responses from varying governments led to different restrictions for each site. This made it difficult to oversee ASCOLT trial operations and monitor changes for each site during the pandemic. For some trial sites, treatment on the study protocol continued while suspending enrollment of new patients. However, for sites in Hong Kong, the study temporarily stopped dispensing oral treatments and delayed trial visits [29].

The Ohio State University Comprehensive Cancer Center (OSUCCC) in Columbus, Ohio, declared a university-wide state of emergency on March 22, 2020, in response to the COVID-19 pandemic [30]. In the days preceding to the announcement, the medical center organized a multipronged central COVID-19 command center. On March 18, 2020, in an effort to limit transmission of the virus, OSUCCC Office of Research released an "Interim Guidance for Human Subjects Research" wherein investigators were urged to limit any human subjects' studies that involved face-to-face contact [31]. Face-to-face visits were either postponed or converted to telemedicine visit when feasible. Furthermore, every human subject study was centrally reviewed by the Office of Research and only "essential" studies were approved for continuation. Investigators were updated through regular virtual town hall meetings organized by the Director and Deputy Director of OSUCCC. From March until August, there were 11 protocol deviations related to COVID-19 for NCTN trials open at OSUCCC. Most deviations were missed appointments, procedures, or exams that were required for the study.

These challenges early in the pandemic led to many operational efficiencies highlighted by the flexibility of many multicenter trials to adapt to a more centralized regulatory process without interrupting research activity $[13,32,33 \bullet]$. In a study collating all recommendations from national agencies for oncology research, the authors recommended that all sponsors and regulatory authorities tolerate "changes to study assessments and interventions that allow individualization of these complex decisions, putting the patient first while continuing to optimize safety and endpoint assessment." [34]

\section{Impact on Research Infrastructure}

The COVID-19 pandemic also directly impacted a shift in research infrastructure at the organization and national level. As hospitals reallocated resources and assets to increase clinical capacity for patient care, there was an additional push towards rapidly developing trial protocols, recruiting collaborators, securing funds, and launching COVID-19 randomized clinical trials. Many of these activities that usually take up to a year were abbreviated to a matter of a few weeks. As the priority for research was shifted towards COVID-19-related activities, many trials were temporarily suspended or decreased accrual [3].

On March 23, 2020, NRG Oncology Leadership reported the epicenter of NRG Oncology Operation had shut down all non-essential businesses with a physical presence and staff shifted to work remotely [12]. In efforts to keep up with many of the regulatory changes, there was an increase in operational burden on trial programs and administrative staff. To centralize the collection of all COVID-19 pandemic-related impact on Alliance clinical trials, on April 15, 2020, the Alliance launched a single Rave Study called ALLIANCE COVID19-Reporting [35]. This simplified process allowed data 
collection on details surrounding withdrawals from treatment or trials due to COVID-19 pandemic, and all pandemic-related protocol deviations on Alliance clinical trials.

Research sites had to deal with both the administrative tasks juxtaposed by a decrease in staff to manage workload [33•]. Regulatory agencies quickly noticed that many trial programs were unable to meet all the protocol-specific requirements and procedures including tumor biopsies, laboratory/diagnostic testing, and imaging [19, 33•]. To mitigate further regulatory burden on specific institutions, the NCI Clinical Trials Monitoring Branch suspended Alliance audits through April 2020 and the Alliance suspended on-site monitoring until July 2020 for clinical trials requiring on-site monitoring [36].

\section{Biobanking}

NCI-funded National Clinical Trials Network Biospecimen Banks are considered essential to oncology research. Due to the COVID-19 pandemic, NCTN biobanks saw a 40\% decrease in receipt of biospecimen [37]. This was primarily due to limited patient enrollment and biospecimen collection at study sites that were prioritizing studies that required real-time analysis of patient samples [18]. There was also uncertainty regarding transmission of virus while handling specimen from patients concurrently infected with SARS-CoV-2 [38, 39]. In addition, the closure of central laboratories temporarily halted biobanking. For example, the ECOG study, EA 1181-"Preoperative THP and Postoperative HP in Patients Who Achieve a Pathologic Complete Response Part 1 Component of: The CompassHER2 Trials (Comprehensive Use of Pathologic Response Assessment to Optimize Therapy in HER2-Positive Breast Cancer)"-- protocol requires tumor blocks to be sent to the ECOG-ACRIN Central Biorepository and Pathology Facility. Due to the need for social distancing of the laboratory staff during the early phases of the pandemic, the ECOG-ACRIN Central Biorepository and Pathology Facility was not accepting samples. Tumor blocks of the clinical biopsy or residual tumors at surgical excision were held at local institutions till further notice [40]. NCTN biobanks also saw a $60 \%$ decrease in distribution of biospecimens [12, 37]. In the beginning of April, SWOG reported that only $58 \%$ of biorepositories $(n=12)$ were accepting specimens as usual [41]. Over the next 2 months, all states began the reopening phases of biobanks, with a few returning to full operation $[37,42]$.

\section{Impact of Social Distancing Measures}

The concept of "nonabandonment" is typically considered in clinical care rather than clinical trials [43••]. However, during the pandemic, balancing the patient's interest in remaining on a potentially curative trial while maintaining appropriate social distancing has posed additional challenges. Overall, cancer patients with COVID-19 have a high 30-day all-cause mortality [44, 45]. Compared to those without cancer, infected cancer patients have a 3.5 times increase risk of requiring ICU admission and ventilation [45]. To maintain social distancing, many NCTN studies adjusted patient visits by reducing the number of follow up imaging, general health measurements, and history and physical collection.

To allow appropriate physical distancing, the NCI approved numerous temporary protocol allowances for cooperative group trials. No formal protocol amendments were made to allow for immediate adoption to the new changes. This ranged from extending the time by 3 or 6 months to the next in-person visit (for exam, blood collection or imaging), conducting the follow-up virtually (telemedicine) if possible, and administering questionnaires through mail, email, or phone. For example, investigators in the Alliance study, Comparison of Axillary Lymph Node Dissection with Axillary Radiation for Patients with NodePositive Breast Cancer Treated with Chemotherapy (NCT01901094), made similar adjustments. Specifically, clinical breast exams were counted as missing if the visit was converted into a telemedicine visit, and a 3-month window was allowed for all follow-up in-person clinic visits and lymphedema assessments if a face-to-face or virtual visit could not be done [46]. Because this trial is also dependent on surgical data, it was impacted by surgical delays that many institutions experienced as a result of the American College of Surgeons COVID-19 guidelines calling for cancellation of elective surgeries [47]. As a result, the investigators of A011202 extended the window from chemotherapy to surgery from 56 to 84 days.

Social distancing measures impacted not only patients entering the healthcare facility but also clinical research coordinators and other trial support staff. Many institutions implemented work from home policies for non-clinical staff, including research support staff [48]. Physical distancing to protect patient and researcher safety has been one of the major aspects that have impeded clinical trials [3]. At Dana Farber, over $75 \%$ of the clinical trials workforce began to work remotely and patients were evaluated by phone or video conference [17]. According to a survey by the American Society of Clinical Oncology, of the 32 survey respondents, including research directors, managers, physician investigators, and research staff, $75 \%$ of the programs mandated remote work for researchers [33•]. Many stated that they implemented remote patient care interactions and remote site initiation visits and monitoring by sponsors or contract research organizations.

\section{Discussion}

The COVID-19 pandemic has had a devasting global impact with over 349,000 deaths in the USA alone reported by the 
CDC in January 2021 [49]. Many aspects of the US healthcare system suffered significant consequences including oncology clinical trials. Even with robust research infrastructure and collaboration, the NCTN will continue to follow flexible regulatory guidance and make the necessary changes to mitigate the impact of this pandemic to current and future clinical trials. As detailed in this paper, the pandemic has generated an opportunity for innovation in clinical trials [13].

\section{Enrollment}

Enrollment into clinical trials has always been a challenge for oncology research where fewer than $10 \%$ of cancer patients in the US enroll [50]. According to the Cancer Research Institute and IQVIA, a human data science company, it is estimated that it will take up to 3-6-month post pandemic before research programs can become "fully" operational again [7]. During the pandemic, the NCTN trials incorporated many alternative approaches including e-signatures for informed consent and virtual patient visits. The nationwide expansion of telemedicine reimbursement during the beginning of the COVID-19 pandemic eased some of this transition. However, as insurance reimbursement and state-wide medical license requirements for telemedicine change and become more stringent, it is unclear if electronic consenting and virtual visits for clinical trial enrollment will be sustained.

\section{Mail Service Program for Medication}

Use of mail service pharmacy in the USA for routine medication continues to grow [51]. The expansion of these services for clinical trial agents have not been widely utilized until the COVID19 pandemic. Distributing medication to homes via a mail service program eliminated the need for at-risk patients to visit trial sites [13, 19, 25]. However, there were limitations in shipping oral CTEP IND agents due to regulatory constraints [25]. Expanding the role of mail order pharmacy to ship oral agents to patients may help reduce the burden of patients participating in clinical trials in the future.

\section{Telemedicine and the Future}

When implementing social distance measures, the appropriate use of telemedicine was a useful strategy for many of the NCTN breast cancer trials during the COVID-19 pandemic. Use of telemedicine allowed not only remote patient care visits but also virtual review of symptoms and adverse events [7, 25, 52]. All NCTN cooperative groups recommended its use, and many providers and patients have positively reacted to the use of telemedicine [13]. Practical challenges remain for ongoing use and expansion in the role of telemedicine. These include lack of training for clinical staff and patients, technology failures, lack of appropriate technology and internet connection for patients, endangerment of patient trust, mismanagement of patient privacy and data management, and inability to perform a full medical exam virtually $[13,53]$. Addressing these limitations will help expand the role of telemedicine in clinical trials.

\section{Conclusion}

The impact of the COVID-19 pandemic will leave long-lasting effects in many aspects of oncology care. The NCI predicted that 10,000 excess cancer deaths will occur over the next decade as a result of missed screenings, delays in diagnosis, and reductions in oncology care caused by the COVID-19 pandemic [54]. In the midst of all the challenges during the COVID-19 pandemic, regulatory agencies, research groups, clinical sites, and individual research teams made impressive strides working in uncertain and resource limiting conditions. An important consideration for future analysis is to conduct longer follow-up to assess the post pandemic effects on clinical trials including protocol deviations, goals, and measurement of outcomes [50]. Given the speed needed for change, there was an immense amount of collaboration and creativity in developing solutions that best fit each trial. The pandemic highlighted aspects of oncology clinical research that could be simplified, modernized, and adapted in order to safely proceed while maintaining research integrity.

\section{Declarations}

Conflict of Interest The authors declare no competing interests.

Human and Animal Rights and Informed Consent This article does not contain any studies with human or animal subjects performed by any of the authors.

\section{References}

Papers of particular interest, published recently, have been highlighted as:

- Of importance

•- Of major importance

1. WHO. WHO Coronavirus Disease (COVID-19) Dashboard. World Health Organization. 2020. https://covid19.who.int/. Accessed 2 Jan 2021.

2. Blumenthal D, Fowler EJ, Abrams M, et al. Covid-19-implications for the Health Care System. N Engl J Med. 2020;383(15): 1483-8.

3. van Dorn A. COVID-19 and readjusting clinical trials. Lancet. 2020;396(10250):523-4.

4. North CM, Dougan ML, Sacks CA. Improving clinical trial enrollment-in the Covid-19 era and beyond. N Engl J Med. 2020;383(15):1406-8.

5. Miller $\mathrm{KD}$, Nogueira $\mathrm{L}$, Mariotto $\mathrm{AB}$, et al. Cancer treatment and survivorship statistics, 2019. CA Cancer J Clin. 2019;69(5):363-85. 
6. Asaad M, Habibullah NK, Butler CE. The impact of COVID-19 on clinical trials. Ann Surg. 2020;272(3):e222-3.

7. Upadhaya S, Yu JX, Oliva C, et al. Impact of COVID-19 on oncology clinical trials. Nat Rev Drug Discov. 2020;19:376-7.

8. Gongora A, Jardim D, Bastos D. Oncology clinical trials during the COVID-19 Pandemic. Oncology. 2020;34:265-9.

9. Park KU, Mamounas EP, Katz MHG, et al. Clinical trials for the surgical oncologist: opportunities and hurdles. Ann Surg Oncol. 2020;27(7):2269-75.

10. Park KU, Weiss A, Francescatti A, et al. What is the national clinical trials network, and how do I join? 2020. https://bulletin.facs. org/2020/03/what-is-the-national-clinical-trials-network-and-howdo-i-join/. Accessed 1 Mar 2020.

11. NCTN accrual for "Intervention" step in trials by Lead Group \& Week: 2-3-2020 to 3-29-2020. OPEN portal system cancer trial support unit. 2020. https://www.swog.org/sites/default/files/docs/ 2020-04/1 st\%20Virtual\%20Joint\%20BSANCAB-2020-04-09JHD-FOR\%20DISTRIBUTION.pdf.

12. NRG Oncology Leadership Special Message Regarding COVID19. NRG Oncology; 2020. https://www.nrgoncology.org/Home/ News/Post/nrg-oncology-leadership-special-messageregardingcovid-19. Accessed 23 Mar 2020.

13. Gensheimer MF, Yom SS, Soto N, et al. Multicenter clinical cancer research after COVID-19: a perspective from NRG oncology. Int J Radiat Oncol Biol Phys. 2020;108(2):483-5.

14. Unger JM, Blanke CD, LeBlanc M, et al. Association of the coronavirus disease 2019 (COVID-19) outbreak with enrollment in cancer clinical trials. JAMA Netw Open. 2020;3(6):e2010651.

15. COVID-19: Data. City of New York. 2020. https://www1.nyc.gov/ site/doh/covid/covid-19-data.page. Accessed 26 Sept 2020.

16. Doroshow J. Clinical Serological Sciences: COVID-19 \& Cancer. National Cancer Institute; 2020. https://deainfo.nci.nih.gov/ advisory/fac/0520/Doroshow.pdf. Accessed 26 Sept 2020.

17. AACR. Clinical research slows as COVID-19 surges. Cancer Discov. 2020;10(5):630.

18. Hall E, Lewis R, Snowdon C. Life after COVID-19 for cancer clinical trials. Int J Radiat Oncol Biol Phys. 2020;108(2):486-8.

19. FDA Guidance on Conduct of Clinical Trials of Medical Products during COVID-19 Public Health Emergency. In: https://www.fda. gov/regulatory-information/search-fda-guidance-documents/fdaguidanceconduct-clinical-trials-medical-products-during-covid-19public-health-emergency, ed.; 2020.

20. Olimid AP, Olimid DA. Ethical review of patient safety and public health in EU clinical trials legislation: impact of COVID-19 pandemic. Romanian J Morphol Embryol. 2020;61(1):277-81.

21. Addis A, Genazzani A, Trotta MP, et al. Promoting better clinical trials and drug information as public health interventions for the COVID-19 emergency in Italy. Ann Intern Med. 2020;173(8): 654-5.

22. Guidance for NIH-funded clinical trials and human subjects studies affected by COVID-19. In: https:/grants.nih.gov/grants/guide/ noticefiles/NOT-OD-20-087.html, ed.; 2020.

23. WHO characterizes COVID-19 as a pandemic. World Health Organization. 2020. https://www.who.int/emergencies/diseases/ novel-coronavirus-2019/events-as-they-happen. Accessed 1 Oct 2020.

24. S1418/BR006 MEMORANDUM - NOTE TO FILE. Southwest Oncology Group Cancer Research Network; 2020. https://www.ctsu. org/readfile.aspx?sectionid=201537. Accessed 26 Sept 2020.

25. Mooney M, McCaskill-Stevens W. Interim guidance for patients on clinical trials supported by the $\mathrm{NCI}$ cancer therapy evaluation program and the NCI community oncology research program; 2020. https://ctep. cancer.gov/content/docs/Memorandum_on_Interim_Guidance for Clinical Trial Activities Affected by the Novel Coronavirus-3-132020.pdf.
26. EA1131 MEMORANDUM protocol-specific deviation guidance for COVID-19 mitigation. ECOG-ACRIN operations office; 2020. https://www.ctsu.org/readfile.aspx?sectionid=202914.

27. Lee C, Mccaskill-Stevens W. Tomosynthesis mammographic Imaging Screening Trial (TMIST): An invitation and opportunity for the national medical association community to shape the future of precision screening for breast cancer. J Natl Med Assoc. 2020;112(6):613-8.

28. EA1151 MEMORANDUM protocol-specific deviation guidance for COVID-19 mitigation. 2020. https://www.ctsu.org/readfile. aspx? sectionid=202918. Accessed 1 Oct 2020.

29. Segelov E, Prenen H, Day D, et al. Impact of the COVID-19 epidemic on a Pan-Asian academic oncology clinical trial. JCO Glob Oncol. 2020;6:585-8.

30. Drake M. Ohio State issues university-wide declaration to manage coronavirus pandemic. OSU State News; 2020. https://news.osu. edu/new-ohio-state-issues-university-wide-declaration-to-managecoronaviruspandemic. Accessed 1 Oct 2020.

31. Pollock R, Shields P. OSUCCC COVID-19 supplemental information for human subject research. The Ohio State University Comprehensive Cancer Center; 2020. http://cancer.convio.net/site/ DocServer/OSUCCC_Human_Subject_Guidance_031820_ FINAL.pdf?docID=9747.

32. Moujaess E, Kourie HR, Ghosn M. Cancer patients and research during COVID-19 pandemic: a systematic review of current evidence. Crit Rev Oncol Hematol. 2020;150:102972.

33. Waterhouse DM, Harvey RD, Hurley P et al. Early impact of COVID-19 on the conduct of oncology clinical trials and long-term opportunities for transformation: findings from an American Society of Clinical Oncology Survey. JCO Oncology Practice $2020 ; 16(7): 417-21$. This study surveyed providers from various settings to describe the major changes and challenges they experienced with respect to cancer clinical trials during early pandemic phase. It highlights the long-term opportunities to improve and transform the clinical trial system.

34. de Paula BHR, Araújo I, Bandeira L, et al. Recommendations from national regulatory agencies for ongoing cancer trials during the COVID-19 pandemic. Lancet Oncol. 2020;21(5):624-7.

35. Alliance COVID-19 data collection memorandum. Alliance statistics and data center: alliance for clinical trials in Oncology; 2020. https:// www.allianceforclinicaltrialsinoncology.org/main/member/cmsfile? $\mathrm{cmsPath}=/ \mathrm{Member} / \mathrm{COVID}-19 /$ files/Memo-COVID19DataCollection-04152020.pdf.

36. Alliance-wide operational considerations for COVID-19 mitigation memorandum. Alliance for Clinical Trials in Oncology; 2020. https://www.allianceforclinicaltrialsinoncology.org/main/member/ cmsfile?cmsPath=/Member/COVID-19/files/Memo-COVID19AllianceOperationalConsiderations-03202020.pdf.

37. Lankes HA, Watson MA, Jordan RC, et al. Abstract PO-065: National Clinical Trials Network biobanking during the COVID19 pandemic. Clin Cancer Res. 2020;26(18 Supplement):PO-065.

38. Hofman P, Puchois $\mathrm{P}$, Brest $\mathrm{P}$, et al. Possible consequences of the COVID-19 pandemic on the use of biospecimens from cancer biobanks for research in academia and bioindustry. Nat Med. 2020;26(6):809-10.

39. Henderson MK, Kozlakidis Z, Fachiroh J et al. The Responses of Biobanks to COVID-19. Biopreservation and Biobanking 2020; 18(6):483-91.

40. EA1181: Protocol-specific deviation guidance for COVID-19 mitigation. Cancer trials support unit: ECOG-ACRIN cancer research group; 2020. https://www.ctsu.org/readfile.aspx? sectionid= 202920.

41. SWOG Cancer Research Network. SWOG Cancer Research Network Laboratory Status. https:/www.swog.org/sites/default/files/docs/ 202004/April\%207\%2C\%202020\%20LAB\%20STATUS.pdf. Accessed 1 Oct 2020. 
42. Shipment of biospecimens held at clinical sites due to COVID-19 biobank restrictions. Alliance for Clinical Trials in Oncology: Cancer Trials Support Unit; 2020. https://www.ctsu.org/readfile. aspx?fname=whatsnew\%2fAlliance-Memo-Shipment-20200815. pdf.

43.• Shuman AG, Pentz RD. Cancer research ethics and COVID-19. Oncologist. 2020;25(6):458-9. Parients with caner enrolled in clinical trials area vulnerabe population. This article discusses major ethical considerations that healthcare providers should consider.

44. Kuderer NM, Choueiri TK, Shah DP, et al. Clinical impact of COVID-19 on patients with cancer (CCC19): a cohort study. Lancet. 2020;395(10241):1907-18.

45. Al-Quteimat OM, Amer AM. The impact of the COVID-19 pandemic on cancer patients. Am J Clin Oncol. 2020;43(6):452-5.

46. Boughey J, Hoffman L. Alliance A011202 protocol-specific minor deviation guidance for COVID-19 mitigation; 2020. https://www. ctsu.org/readfile.aspx?sectionid=201342.

47. COVID-19 guidelines for triage of breast cancer patients. American College of Surgeons; 2020. https://www.facs.org/covid-19/ clinicalguidance/elective-case/breast-cancer.

48. Tolaney SM, Lydon CA, Li T et al. The impact of COVID-19 on clinical trial execution at the Dana-Farber Cancer Institute. J Natl Cancer Inst. 2020. https://doi.org/10.1093/jnci/djaa144.
49. Provisional death counts for coronavirus disease 2019 (COVID19). Centers for disease control and prevention; 2020. https:// www.cdc.gov/nchs/nvss/vsrr/covid19/index.htm. Accessed 29 Sept 2020.

50. Nabhan C, Choueiri TK, Mato AR. Rethinking clinical trials reform during the COVID-19 pandemic. JAMA Oncology 2020;6(9): 1327.

51. Suda KJ, Hicks LA, Roberts RM, et al. Antibiotic expenditures by medication, class, and healthcare setting in the United States, 2010 2015. Clin Infect Dis. 2017;66(2):185-90.

52. Marcum M, Kurtzweil N, Vollmer C, et al. COVID-19 pandemic and impact on cancer clinical trials: an academic medical center perspective. Cancer Med. 2020;9(17):6141-6.

53. De Las Heras B, Saini KS, Boyle F et al. Cancer treatment and research during the COVID-19 pandemic: experience of the first 6 Months. Oncology and Therapy 2020;8(2):171-82.

54. Sharpless NE. COVID-19 and cancer. Science. 2020;368(6497):1290.

Publisher's Note Springer Nature remains neutral with regard to jurisdictional claims in published maps and institutional affiliations. 